\section{COVID-19 could be the most frequent occupational disease in Romania in 2020}

\author{
Marina Ruxandra Oțelea ${ }^{1}$, Oana Cristina \\ Arghir ${ }^{2,3}$, Raluca Constantin ${ }^{4}$, Agripina Rașcu ${ }^{1,5}$ \\ 'University of Medicine and Pharmacy "Carol Davila", \\ Clinical Department 2, Bucharest, Romania \\ 2 Faculty of Medicine, "Ovidius" University of Constanța, \\ 4th Department of Clinical Diseases, Romania \\ ${ }^{3}$ Clinical Pneumophthisiology Hospital, Constanța, \\ Romania \\ ${ }^{4}$ St Andrew Clinical County Emergency Hospital, \\ Constanta, Romania \\ ${ }^{5}$ Clinical Hospital Colentina, Clinic for Occupational \\ Diseases, Bucharest, Romania
}

Corresponding author

Marina Ruxandra Oțelea

marina.otelea@umfcd.ro

\begin{abstract}
The definition of COVID-19 as occupational disease follows the investigation of any other occupational disease caused by an infectious agent. The risk is not equal for all occupations and the occupational physician has to assess the working conditions to conclude a diagnosis of occupational COVID-19. In the pandemic context, employees face also other occupational hazards. The high level of work load and the scarce resources lead to stress, physical and mental exhaustion and irregular sleep. The protection measures, of undisputable benefit, increase the risk for contact dermatitis. There is a high probability for medium and possible long term effects of COVID-19, such as the post-traumatic stress disorder or the respiratory sequelae. These consequences need to be acknowledged and properly manged by the medical team taking care of the patient. This review presents the main characteristics of the occupational related disorders during and after the current pandemia.
\end{abstract}

Keywords: COVID-19, occupational stress, burnout, contact dermatitis, post traumatic syndrome, lung damage post COVID-19

\section{Introduction}

COVID-19 pandemic is currently the number one public health threat and issue and the most prevalent occupational disease in the healthcare area worldwide. It has been diagnosed in 3,300 healthcare workers in China (3,835 of the total cases) [1] and among almost $20 \%$ of the Italian healthcare personnel [2]. Romania has already reported 812 COVID-19 cases in healthcare workers, representing $12.8 \%$ of the total
Romanian cases confirmed until the 4th of April. The number continued to grow afterwards, as was the number of registred cases in the communities. The rate of death among the healthcare workers was $0.24 \%(n=2 / 812)$ [3]. Obviously, the first priority of healthcare system is preventing the occurrence of new cases and recommending the most appropriate cure of the existing therapies, without ignoring to identify the features of this new occupational disease. 


\section{The COVID-19 infection}

The etiology of COVID-19 is well established. SARSCOV-2 is an RNA virus, a new member of the beta class of coronaviruses. This class of coronaviruses includes 2 other strains that caused severe human respiratory epidemics in the last decade, SARS and MERS. SARSCOV-2 multiplies primarily in the respiratory tract and binds to the ACE-2 receptor of the epithelial cells. SARS-COV-2 has a higher affinity for ACE-2 receptor than SARS-COV-1. This characteristic augments its capacity of interhuman transmission [4].

There are three routes of SARS-COV-2 transmission: by droplets from coughing and sneezing, the most contagious way, by touch (direct contact) and by aerosol transmission [5]. Sporadic cases of transmission from asymptomatic carriers [6] and a potential fecal-oral route were raised [7]; for the latest route, the conclusive evidence is missing. The susceptibility to infection is quasi-universal, as this novel virus has a high tropism for human species and there is no immune memory to work on its defence. The reasons for the frequently mild cases in children are not yet known.

In general, the contagiousness of any infection is estimated by the basic reproduction number (Ro). The Ro is the measure of the rapidity of the spread of the disease within the population, estimated by the number of infected persons arising from a single contact case. Reports from China outbreak showed a Ro for SARS-COV-2 of $2.13-3.33$ [8] that is twice higher than the Ro for the seasonal flu, but more than 4 times lower than Ro for measles [9].

The healthcare force is not the only population at risk: any employee that has been in close face-to-face contact with a client, infected with SARS-COV-2, could claim an occupational source of the disease. However, there is, definitely, a higher risk for working in emergency units, medical departments, but, also, in police and firefighter squads, in public transportation, in the delivery of goods, or the cleaning services [10]. The clinical picture of COVID-19 is dominated by fever, malaise and respiratory symptoms (cough, dyspnoea and sputum production, hemoptysis being a rare finding) [9]. Diarrhea is present in $2-50 \%$ of the cases [7]. More rarely encountered symptoms are headache or dizziness (12\%), nausea and vomiting (3.9\%) [11]. The majority of cases are mild to moderate, but acute respiratory distress syndrome, septic shock, cytokine release syndrome and multi organ failure might develop, significantly raising the fatality rate [1].

A meta-analysis of the China epidemics reports showed a fatality rate of 5\% [11]. This figure is quite diverse for the countries in Europe, ranging from $11.9 \%$ in Italy to $1.2 \%$ in Germany. This wide variation largely reflects the number of tests performed inside the population and the rapidity of social distance and mitigation measures that were enforced [12].

\section{The occupational disease diagnosis}

Due to the high risk of exposure for the healthcare workers at to the COVID-19, there is already a shortage of qualified professionals to fight against this disease. World Health Organization (WHO) has issued a procedure of risk assessment for SARSCOV-2 exposure. The management of this risk covers questions related to possible household, travel and work contamination [13].

Briefly, the WHO document states that the risk assessment should include the occupation, the type of health care facility and the number and types of interactions or procedures performed on COVID-19 patients. If a healthcare worker had a face-to-face contact, performed an aerosol-generating procedure or had contact with the contaminated environment from a COVID-19 patient, he should include in the exposed category of workers. The risk assessment is largely dependent on the degree of safety measures that are taken and on the adherence to these measures. If there is any answer indicating that the personal protective equipment (including the protocol of replacement), the hand hygiene, the surface decontamination procedures were not always followed as recommended, the person is included in the high category risk. If the adherence to preventing the SARS CoV2 infection is high, but procedures generating aerosols are recommended, or there is a splash of fluid from a COVID-19 patient, the worker is, also, at high risk of exposure.

In terms of the Romanian recommendations, these are far less detailed. The National Institute of the Public Health states that only the healthcare worker "that wore the standard individual protection equipment (mask, gloves, face shield or goggles/ protective glasses for those performing procedures that generate aerosols) are not considered a close contact"[14].

Given the specificities related to the possible routes of SARS CoV2 transmission and from the point of view of standard occupational disease investigation and diagnosis, COVID-19 is an infectious disease with a high rate of occupational exposure. It is important to clearly consider the occupational transmission, because not all COVID-19 cases in healthcare 
workers are of occupational origin. The communitytransmitted viral infection requires a detailed history to differentiate the occupational from the nonoccupational acquired disease.

The political measures to ensure compensation for all medical personnel during this pandemic, particularly in those that are at high risk to become infected, should not substitute the declaration of COVID-19 as an occupational disease, because one of the main purposes of this recognition means more prevention and better control policy. The emergency state imposes quick measures. Therefore, each case should become a source of risk identification and improvement of the medical procedures whenever necessary.

\section{The specificity of this new occupational disease}

Meanwhile, there are still some specificities of the occupational COVID-19 disease that probably should be classified in a broader category of SARS-COV-2 related diseases. Until now, at least 2 entities should be discussed: the burnout syndrome and the occupational dermatitis.

The occupational burnout syndrome is an emotional and physical exhaustion related to an increased demand and/or inadequate resources.The occupational burnout syndrome is an emotional and physical exhaustion related to an increased demand and/or inadequate resources. These occupational risk factors (both increase demand and inadequate resources) are THE typical work conditions during a pandemic outbreak. The topic is of a high interest for preserving the individual health but also for maintaining the quality of healthcare and the avoidance of the medical errors [15].

The initial tool designed for the definition of burnout was the Maslach Burnout Inventory. This questionnaire included 3 subscales: "emotional exhaustion", "depersonalization" and "personal accomplishment", which were maintained in the 11th revision of the WHO-International Classification of Diseases definition of the syndrome [16]. The initial questionnaire was developed for research purpose and further on variants of it were developed, such as the Oldenburg Inventory, the Copenhagen Burnout Inventory) [17,18] adding new or subtracting/ replacing subscales. Recently new conceptual frames were developed adding elements from the general health questionnaire, cognitive defects [19] or even proposing an organizational approach [20].
The extreme physical exhaustion from work overload and stress is a component of the Karoshi syndrome covering both the cerebro-vascular disease or ischemic heart disease and the suicide from overwork. [21] As the overwork increases the level of catecholamines, the raised blood pressure [22] creates a state of hypercoagulability [23] and predispose to life threatening arrhythmia [24,25]. So, the ambulatory monitoring for heart rhythm and blood pressure [26] could be useful for the early diagnosis and the prevention of the severe physical outcomes of burnout.

Currently, there are few published data on the mental impact of healthcare workers treating patients with COVID-19, requiring proper assessment and management of cases and lessons from previous epidemics might help. Nurses in areas with high number of cases and front line healthcare workers, developed more symptoms of depression and anxiety and higher scores of insomnia severity index [27, 28] similar with those developed during the SARS1 epidemic [29]. The number of working hours, anxiety and being the only child in the family were closely correlated to the stress level in nurses. [30]

Beside the overload and scarce resources, there are many changes in the medical procedures and in the medical delivery of services: telemedicine is increasing, recommendations for chronic diseases is are differently prioritized, routine interventions are postponed and different medical societies have temporary updated their recommendations for the medical care $[31,32,33]$.

The relation between stress and infection is far from reaching a consensus and conclusive evidence. However, a certain contribution of stress to vulnerability to infection was emphasized [34] and a better emotion management and resilience was found to be protective [35].

There are also several guidelines developed in order to ensure a good management [36] (or to assist healthcare workers [37] and these interventions that mitigate stress should be implemented.

\section{Occupational dermatitis}

It is well known that medical personnel, particularly nurses and doctors, have an increased risk of wet dermatitis [38]. In normal circumstances, a limit of $<2 \mathrm{~h} /$ day of wet environment exposure and < 20 hand washes/shift are recommended [39,40], but in the current situation these thresholds could be easily exceeded for safety reasons. In order to avoid contact transmission hand hygiene should be done before touching a patient or performing an invasive procedure, after potential exposure 
to patient's fluids or after touching possible contaminated surface or the patient. These rules apply to all, no matter if wearing gloves or not.

Usage of disinfectants or detergents and prolonged occlusion with gloves will make the hyperemia more quickly visible. In fact, gloves wearing will raise the superficial $\mathrm{pH}$ of the skin, impairing even more the cutaneous barrier [41]. A systematic review showed that the skin barrier impairment caused by detergents/soaps increases with the duration of gloves wearing [42]. Skin occlusion (due to gloves) stimulates the absorption of non-polar liposoluble substances and amplifies the irritative effect of some substances (e.g. urea) [43].

In a Wuhan survey, $74.5 \%$ of the respondents reported adverse skin reactions. In order of the frequency (from highest to lowest) these symptoms were: dryness, papules, erythema, maceration of the hands, cheecks and nasal bridge [44].

In order to prevent dermatological disorders, moistures and gels should be applied before the protective equipment is used.

Maceration should be revealed (exposed to open air) or treated with boric acid solution $3 \%$, normal saline or zinc oxide ointment. If contact dermatitis occurs, corticoid treatment is recommended. A complete description of the hand hygiene, the skincare measures after long term wearing of gloves, the prevention of pressure injury caused by masks and goggles, the treatment of delayed pressure urticaria, contact dermatitis and other dermatological conditions is available in the consensus published by Yan et al in a recent number of Dermatologic Therapy [45].

\section{Is there a post- Covid-19 syndrome?}

Many voices underline that life will change in various aspects after this unprecedented challenge humanity passes through. We don't know this, but as far as healthcare will be concerned, we can make some reasonable assumptions.

The stressful working conditions will not end when the epidemic will naturally come down. The opening of the wards for both acute and chronic patients will create further burden on medical personnel. The postponed elective surgeries [32,33] will increase the number of surgical procedures in the following period of time. It is, also, possible that, in some cases, the postponed intervention will increase the severity of the disease. This will require more complex therapeutic interventions.

The suddenly increase in telemedicine consultations pushed doctors and patients to change behavior. It will be a challenge for medical professionals to further define how to continue this new type of encounter, in which categories of patients or circumstances to be applied, yet the gained experience during this stressful period should be further evaluated. The decisions for the reimbursement of services made by insurance companies will influence the continuation of a "time saving" pattern of work for both medical providers and patients.

From previous epidemics, compared to patients, healthcare workers confronting contagious diseases had a significantly higher levels of stress, anxiety, depression and posttraumatic stress one year after the outbreaks [46]. Access to knowledge about the natural history of a disease, a positive coping attitude and social support are key elements for a better outcome [47].

In terms of occupational medicine a post-traumatic syndrome might be diagnosed in some patients suffering from occupational COVID-19 that may affect their short and medium term fitness for work and social integration.

The long-term lung damage postCOVID-19 is not yet defined but suspected, because similar fibrosis scaring lesions have been documented in SARS and MERS survivors $[48,49]$. The persistent lung abnormalities on CT scan after the discharge from hospital are a red flag for a future chronic lung disease. So, longterm studies of follow-up investigations are needed $[49,50]$ and occupational physicians together with pulmonologists should monitor the lung function and the work for an early diagnosis of these complications.

\section{Conclusions}

The current COVID-19 pandemic will provide the greatest number of occupational diseases during this year and not only in healthcare workers. Occupational physicians hope that administrative procedures will not impede the recognition of occupational exposure and keep open the dossier of reconsidering the occupational significance in general.

Today, when the main focus is on prevention in any workplace and community if necessary the occupational medicine team should provide guidance to employers and employees in accordance with the international and national regulations.

Long-term health consequences of COVID-19 are not well known. When the wave of the COVID-19 pandemic will pass, we have to continue the follow up its consequences, to provide screening for stress related disorders, for functional status of 
the respiratory disorders and to ensure the proper management of the cases.

\section{References}

1. Wang, Y, Wang, Y, Chen, Y, Qin, Q. Unique epidemiological and clinical features of the emerging 2019 novel coronavirus pneumonia (COVID-19) implicate special control measures. J Med Virol. 2020; 1-9.

2. The Lancet. Editorial. 2020: 395(10228):922.

3. National Institute of Public Health. http://www.cnscbt.ro/index. $\mathrm{php} /$ home.

4. Davenne E, Giot JB, Huynen P. Coronavirus and COVID-19 : focus on a galopping pandemic. Rev Med Liege. 2020;75(4):218-225.

5. Rothan HA, Byrareddy SN. The epidemiology and pathogenesis of coronavirus disease (COVID-19) outbreak. J Autoimmun. 2020;109:102433.

6. Bai Y, Yao L, Wei T, et al. Presumed Asymptomatic Carrier Transmission of COVID-19. JAMA, 2020. doi:10.1001/ jama.2020.2565.

7. D’Amico F, Baumgart DC, Danese S, Peyrin-Biroulet L4. Diarrhea during COVID-19 infection: pathogenesis, epidemiology, prevention and management. Clin Gastroenterol Hepatol. 2020 Apr 8. pii: S15423565(20)30481-X. doi: 10.1016/j.cgh.2020.04.001.

8 7.han S, I.in O, Ran .J, Musa SS, Yang G, Wang W, et al. The basic reproduction number of novel coronavirus (2019-nCoV) estimation based on exponential growth in the early outbreak in China from 2019 to 2020: A reply to Dhungana. Int J Infect Dis (2020),doi: https://doi.org/10.1016/j.ijid.2020.02.025

9. Lake MA. What we know so far: COVID-19 current clinical knowledge and research. Clin Med (Lond). 2020;20(2):124-127.

10. Sim MR. The COVID-19 pandemic: major risks to healthcare and other workers on the front line. Occup Environ Med 2020. doi: 10.1136/oemed-2020-106567.

11. Li LQ, Huang T, Wang YQ, Wang ZP, Liang Y, Huang TB et al. COVID-19 patients' clinical characteristics, discharge rate, and fatality rate of meta-analysis. J Med Virol. 2020; 1- 7.

12. Stafford N. Covid-19: Why Germany's case fatality rate seems so low. BMJ. 2020;369:m1395.

13. Risk assessment and management of exposure of health care workers in the context of COVID-19. https://apps.who.int/iris/ bitstream/handle/10665/331496/WHO-2019-nCov-HCW_risk_ assessment-2020.2-eng.pdf.

14. Definitiile de caz pentru Sindromul respirator acut cu noul coronavirus (COVID-19). Actualizare 27.03.2020. http://www.cnscbt. ro/index.php/informatii-pentru-personalul-medico-sanitar/1594definitia-de-caz-covid-19-actualizare-27-03-2020/file

15. West, CP, Dyrbye, LN, Shanafelt, TD. J Intern Med 2018; 283: 516- 529.

16. WHO. ICD-11. International classification of diseases 11th Revision. The global standard for diagnostic health information. https://www.who.int/classifications/icd/en/

17. Demerouti E, Bakker A. B., Nachreiner F, Schaufeli WB. The job demands-resources model of burnout. J. Appl. Psychol. 2001; 86: 499-512.

18. Kristensen TS, Borritz M, Villadsen E, Christensen KB. (2005). Copenhagen Burnout Inventory (CBI) [Database record]. APA PsycTests.

19. Tavella G, Hadzi-Pavlovic D, Parker G. Burnout: Re-examining its key constructs. Psychiatry Res. 2020:287:112917.

20. Doulougeri K, Georganta K, Montgomery A. Diagnosing” burnout among healthcare professionals: Can we find consensus?. Cogent Med; 2016; 3:1.

21. Eguchi H, Wada K, Smith DR. Recognition, Compensation, and Prevention of Karoshi, or Death due to Overwork. J Occup Environ Med. 2016:58(8): e313-e314.

22. Ke DS. Overwork, stroke, and karoshi-death from overwork. Acta
Neurol Taiwan. 2012:21(2):54-9.

23. Otsui K, Yamamoto J, Inoue N. Overwork accelerates thrombotic reaction: implications for the pathogenesis of Karoshi. J Thromb Thrombolysis. 2018;45:222-224.

24. Wong CW, Chan YH, Cheng YH, Lam CS.Is overwork a precipitant factor of idiopathic ventricular fibrillation? Int J Cardiol. 2016; 223:218- 219 .

25. Xiao N, Yang BF, Shi JZ, et al. Karoshi May Be a Consequence of Overwork-Related Malignant Arrhythmia. Med Sci Monit. 2019;25:357-364

26. Lo EV, Wei YH, Hwang BF. Association between occupational burnout and heart rate variability: A pilot study in a high-tech company in Taiwan. Medicine (Baltimore). 2020;99(2):e18630.

27. Zhang C, Yang L, Liu S, Ma S, Wang Y, Cai Z, et al. Survey of Insomnia and Related Social Psychological Factors Among Medical Staff Involved in the 2019 Novel Coronavirus Disease Outbreak. Frontiers in Psychiatry. 2020;11:306 .

28. Ma S, Wang Y, et al. Factors Associated With Mental Health Outcomes Among Health Care Workers Exposed to Coronavirus Disease 2019. JAMA Netw Open. 2020;3(3):e203976.

29. Maunder R, Hunter J, Vincent L, Bennett J, Peladeau N, Leszcz $\mathrm{M}$, et al. The immediate psychological and occupational impact of the 2003 SARS outbreak in a teaching hospital. CMAJ. 2003;168(10):1245 $-1251$.

30. Mo Y, Deng L, Zhang L, Lang Q, Liao C, Wang N, et al. Work stress among Chinese nurses to support Wuhan for fighting against the COVID-19 epidemic. J Nurs Manag. 2020;Apr 7. doi: 10.1111/ jonm.13014.

31. American Stroke Association. Temporary Emergency Guidance to US Stroke Centers During the COVID-19 Pandemic. Stroke. 2020 Apr 1. doi:10.1161/STROKEAHA.120.030023.

32. Ross RW, Lauer CW, Miles WS, Green JM, Britton Christmas A, May AK, Matthews BD. Maximizing the Calm Before the Storm: Tiered Surgical Response Plan for Novel

Coronavirus (COVID-19). J Am Col Surg 2020 doi: https://doi. org/10.1016/j.jamcollsurg.2020.03.019

33. Puliatti S, Eissa A, Eissa R, Amato M, Mazzone E, Dell'Oglio P, et al. COVID-19 and Urology: A Comprehensive Review of the Literature. BJU Int. 2020 Apr 6. doi: 10.1111/bju.15071.

34. Dhabhar FS. Effects of stress on immune function: the good, the bad, and the beautiful. Immunol Res.2014;58:193-210.

35. Brown RL, Shahane AD, Chen MA, Fagundes CP. Cognitive reappraisal and nasal cytokine production following experimental rhinovirus infection. Brain, Behavior, \& Immunity - Health. 2020;1:100012.

36. Petzold MB, Plag J, Ströhle A. Dealing with psychological distress by healthcare professionals during the COVID-19 pandemia. Nervenarzt. 2020;1-5

37. World Health Organization, War Trauma Foundation and World Vision International (2011). Psychological first aid: Guide for field workers. WHO: Geneva.

38. Meding B, Anveden Berglind I, Alderling M, Lindahl G, Wrangsjö K. Water exposure - challenging differences between occupations. Contact Dermatitis. 2016: 74: 22-28.

39. Australian Government. Australian safety and Compensation Council. Guidance on the prevention of dermatitis caused by wet work. ISBN 0642325308.

40. Flyvholm MA, Lindberg M. OEESC-2005 -- Summing up on the theme Irritants and Wet Work. Contact Dermatitis. 2005;55:317321.

41. Jungersted JM, Scheer H, Mempel M, Baurecht H, Cifuentes L, Høgh JK, et al. Stratum corneum lipids, skin barrier function and filaggrin mutations in patients with atopic eczema. Allergy; 2010; 65: 911-918.

42. Tiedemann D, Clausen ML, John SM, Angelova-Fischer I, Kezic S. and Agner T. Effect of glove occlusion on the skin barrier. Contact Dermatitis. 2016; 74: 2-10.

43. Agner T. An experimental study of irritant effects of urea in different vehicles. Acta Derm Venereol Suppl (Stockh) 1992: 177: 
44-46.

44. Lin P, Zhu S, Huang Y, Li L, Tao J, Lei T, et al. Adverse Skin Reactions Among Healthcare Workers During the Coronavirus Disease 2019 Outbreak: A Survey in Wuhan and Its Surrounding Regions. Br J Dermatol. 2020 Apr 7. doi: 10.1111/bjd.19089.

45. Yan Y, Chen H, Chen L, Cheng B, Diao P, Dong L, et al. Consensus of Chinese experts on protection of skin and mucous membrane barrier for health-care workers fighting against coronavirus disease 2019. Dermatol Ther. 2020 Mar 13:e13310. doi: 10.1111/dth.13310 46. Lee AM, Wong JG, McAlonan GM, et al. Stress and psychological distress among SARS survivors 1 year after the outbreak. Can J Psychiatry. 2007;52(4):233-240).

47. Su TP, Lien TC, Yang CY, et al. Prevalence of psychiatric morbidity and psychological adaptation of the nurses in a structured SARS caring unit during outbreak: a prospective and periodic assessment study in Taiwan. J Psychiatr Res. 2007;41(1-2):119-130

48. Das KM, Lee EY, Singh R, Enani MA, Al Dossari K, Van Gorkom K, et al. Follow-up chest radiographic findings in patients with MERSCoV after recovery. Indian J Radiold Imaging. 2017(3):342-349.

49. Zhang P, Li J, Liu H, Han N, Ju J, Kou Y et al. Long-term bone and lung consequences associated with hospital-acquired severe acute respiratory syndrome: a 15-year follow-up from a prospective cohort study. Bone Res. 2020;8: 8. https://doi.org/10.1038/s41413020-0084-5

50. Wu J, Pan J, Teng D, Xu X, Feng J, Chen YC. Interpretation of CT signs of 2019 novel coronavirus (COVID-19) pneumonia. Eur Radiol. 2020;1-8. doi:10.1007/s00330-020-06915-5. 